\title{
AN INFERENTIAL ACCOUNT ON THEORETICAL CONCEPTS IN PHYSICS
}

\author{
JAVIER ANTA \\ Universitat de Barcelona \\ LOGOS \\ BIAP \\ antajav@gmail.com
}

SUMMARY: In this paper we develop an inferential account on the meaning and reference of theoretical concepts in physics, mainly based on the pragmatic notion of 'inferential validity'. Firstly, we distinguish between empirical meaningfulness and theoretical significance as two different modes of meaning, wherein the former depends on consistently encoding experimental values, as proposed by Chang (2004), and the latter on being semantically coherent with other concepts. Secondly, we argue that each of these contributions to the validity of inferences imports a causal and representational mechanism of reference-fixing, respectively. Finally, we will rely on entropy concepts as our case study.

KEY WORDS: scientific concepts, inferences, entropy concepts, thermal physics, reference

RESUMEN: En este artículo desarrollaré una propuesta inferencial acerca del sentido y referencia de conceptos en física, basada principalmente en la noción pragmática de "validez inferencial". Primero, distinguiremos entre sentido empírico y significado teórico como dos modalidades diferentes del sentido, donde el primero depende de la codificación consistente de valores experimentales, en la línea de Chang (2004), y el segundo en la coherencia semántica con otros conceptos. Además, argumentaremos que estas distintas contribuciones a la validez de las inferencias conllevan mecanismos de fijación referencial causales y representacionales-descriptivos, respectivamente. Finalmente, nos basaremos en los conceptos de entropía como caso de estudio de nuestra propuesta.

PALABRAS CLAVE: conceptos científicos, inferencias, entropía, termofísica, referencia

\section{Introduction}

The problem of how the concepts that emerge within scientific theories and practices acquire their meaning has been central to the whole history of the philosophy of science. Both in logical positivism, wherein concepts were mere syntactic theoretically-independent nodes in a formal-deductive web, and in the historicist philosophies of Kuhn and Feyerabend, for whom the content of concepts depended on their place within theories, the analysis of how con- 
cepts acquire empirical-theoretical meaning and come to refer to their respective phenomena has been a merely subsidiary issue to the logical reconstruction of theories or to the narrative of the historical evolution of scientific disciplines, respectively. ${ }^{1}$ This lack of attention changed substantially within the last decades, when the role of theoretical $^{2}$ concepts in various scientific practices (e.g. measurement processes, phenomena modeling, predictive procedures, and so on) acquired enormous philosophical relevance.

One of the main trends in the current philosophical assessment of scientific concepts is to explain their theoretical (as well as empirical) meaning and referential effectiveness from historical and cognitive factors, emphasizing their role in the development and use of scientific models in actual practices. ${ }^{3}$ In this manifold of pragmatic views, Peacocke (1992) defined scientific concepts as the cognitive acceptance of a set of inferential rules. Years later, Wilson (2006) also developed a complex perspective that treats theoretical concepts in the natural sciences as inferential connections that vary dynamically depending on their application context.

In this paper I will depart from Peacocke's and Wilson's proposals to develop an inferential conception of theoretical concepts in the context of the physical sciences, which would mainly affirm that these should be understood by the way in which their content contributes to the inferential obtaining of relevant information about reality from scientific representations. Our main thesis will be that the semantic content or meaning of theoretical physical concepts can be differentiated between two main ways in which they contribute to an inference being 'valid', namely: their empirical meaning, connected to experimental measurement practices, and their theoretical significance, linked to the semantic coherence of concepts in a representation. Therefore, central to this proposal will be the (epistemic-pragmatic rather than logical $)^{4}$ notion of 'inferential validity', which we can initially define as the fact that the information that has been inferred

${ }^{1}$ See Kindi and Arabatzis 2008, pp. 348-352.

2 As Andreas (2017) points out, the term 'theoretical concept' has two remarkably different understandings. On the one hand, the term 'theoretical' points out that the entity or property to which the concept refers is not observable, which according to the logical-empiricist criterion of Carnap (1966) and his heirs means that such entity or property is not directly perceptible. On the other hand, the term 'theoretical' would refer (especially within the philosophies of historicist science) to the semantic dependence of the concept on a given theory.

\footnotetext{
${ }^{3}$ See Nersessian 2008.

${ }^{4}$ This means that the notion of validity will not only apply to the truth-preserving deductive inferences of classical logic, but to any kind of inferences (abductive,
} 
depends semantically and syntactically on the content of the conceptual resources of a representation. Once our inferential proposal has been exposed, I will use it to clarify how the content of the concept (or better said 'concepts') of entropy works within thermal physics, delimiting in what sense it could be empirically meaningful and/or thermophysically significant and how these modes of meaning allow us to obtain relevant information about thermophysical phenomena.

The plan for this paper is the following. In the next section we will explore two of the most relevant current positions on theoretical concepts, mainly Chang's neo-operationalism (2004) and Nersessian's historicist cognitivism (2008). In section 3 we will develop our inferential account on the meaning of theoretical concepts in physics, distinguishing between empirical meaningfulness and theoretical significance as different kinds of contributions to the validity of inferences within actual practices. Additionally, section 4 will be devoted to understanding in which sense the empirical meaningfulness and the theoretical significance of concepts can provide causal and representational reference-fixing mechanisms. Finally, we will apply this inferential framework to the particular case of entropy concepts as a case study.

\section{Current Views on Theoretical Concepts: Neo-Operationalism and Historical-Cognitivism}

Systematic philosophical assessment of theoretical concepts, not in general but in the particular field of the natural sciences, extends throughout the twentieth century to the present day. There is no doubt that one of the most historical and currently relevant perspectives is what is known as 'operationalism'. Operationalism broadly defends that the meaning of scientific concepts might be completely fixed by a specific set of measurement operations (although the nature and characterization of these operations is debatable) by which the content of this concept can be completely defined. This view was originally developed by the physicist Percy Bridgman (1927) during a period ranging from the 1920s to the 1950s, and was quickly assimilated by the Vienna Circle and its heirs.

In this context, Carnap argued that the notion of 'concept' should be rejected in favor of that of 'term' due to the psychological connotations of the former. Within this philosophical framework, the content of theoretical concepts (which are inserted within primitive

inductive, probabilistic, causal, etc.) that an epistemic agent can realistically perform in a practical context. 
statements or hypotheses of a given theory) depends on their being formally connected within the conceptual network of that theory with its empirical infrastructure made up of empirically measurable statements. That is, according to the proto-operationalist Carnapian proposal, the meaning of a theoretical concept is syntactically and deductively derived from a set of observable statements formulable from measurement operations, without the semantic content of such statements playing any relevant role in such process. The concepts are nothing but nodes in the deductive network that conforms the structure of a particular scientific theory (Kindi and Arabatzis 2008. p. 348).

One of the main defenses of operationalism in the current philosophy of science was carried out by Hasok Chang (2004) in his celebrated Inventing Temperature, where he traces the genesis of the concept of temperature through the historical evolution of the thermometric discipline. However, his neo-operationalist proposal radically departs from previous logical-empirical versions by understanding operations not as structural sets of idealized observational statements but as real measurement practices. For Chang, the significant physical meaning of "KELVIN TEMPERATURE" (developed by Lord Kelvin in the late 1840s) depends intrinsically on the consistency of thermometric techniques and operations employed by the scientific community in a particular practical context. In particular, the semantic content of this thermophysical concept is based on the theoretical-technical possibility of using absolute temperature scales, à la Kelvin or Rankine, in actual measurement procedures.

For what we can call 'reductionist operationalism', the meaning of scientific concepts would be fixed independently of the theory in which they are inserted, precisely because the different measurement operations are not intrinsically associated with certain theories. All that is required is that the theoretical systems 'touch the observational ground"6 (Chang 2019). In this sense, reductionist operationalism would defend that the content of concepts is operationally fixed, and therefore theoretically independent or constant across different theoretical contexts: the meaning of the concept of absolute temper-

\footnotetext{
${ }^{5}$ Henceforth, we will use the convention of using capital letters (e.g. 'HEAT') for denoting concepts.

${ }^{6}$ Actually, Chang (2004) pointed out in detail how the development of certain thermometric practices in the first half of the 19th century was clearly independent of all pre-existing theories on temperature and heat; which suggests that the historical evolution of theoretical concepts is not correlated with the evolution of measurement operations.
} 
ature is the same both within the theory of heat (whether historically rejected or not) and in phenomenological thermodynamics. This applies not only to those concepts that are directly connected with the operational basis, as is the case with 'KELVIN TEMPERATURE', but also to those that are connected by other concepts as is the case with 'CLASIUS ENTROPY', defined by ratios of infinitesimal variations of heat quantities ' $Q$ ' and values of absolute temperature quantities ' $T$ ' of a system.

The theoretical independence of concepts underlying operationalism has straightforward consequences for the problem of concept identification. For example, 'KELVIN TEMPERATURE' and 'SPECIFIC TEMPERATURE' would constitute two separate empirical concepts if each is semantically connected with distinct sets of thermometric operations. However, for those concepts whose content is not directly observable, such as entropy in our case, the situation is more complex. According to operationalism the only element that contributes to meaning is the connection of a concept with the operational basis, two terms such as 'CLASIUS ENTROPY' and 'BOLTZMANN ENTROPY' form a single concept since both depend on the same set of thermometric operations (those linked to 'KELVIN TEMPERATURE'), despite the fact that the two former concepts are theoretically embedded within different theories such as thermodynamics and statistical mechanics, respectively. Another major disadvantage is the inability to distinguish between concepts as such and the mental access of scientific agents to the content of these concepts, both of which are made possible by operational definitions. Chang (2019) discusses the problematic nature of understanding operations (Bridgman (1927) himself does not make it clear how the notion of 'operation' is to be understood in detail) not only as measurement activities but also as mental processes. However, extending the notion of 'operation' to the mental domain does not illuminate in any way the conditions under which an agent possesses a given scientific concept.

Another of the most relevant current philosophical perspectives is Nersessian's cognitive-historical proposal (2008). This author retrieves part of the approach from the historicist tradition of Kuhn and Feyerabend, emphasizing the analysis of real historical cases and rejecting decontextualized evaluations of the conduct of science, adding certain features of the methodology of cognitive sciences to study such as the epistemic dynamics of scientific agents in concrete scientific practices. From this historical-cognitive method, scientific concepts cannot be properly formed from the traditional dichotomy between theoretical elements and observational elements. In fact, 
Nersessian's proposal can be found in the orbit of Shapere (1985), who defended against the historicist currents of the moment that concepts are 'trans-theoretical' entities and therefore their content does not depend on the theory in which they are inserted. For Nersessian, concepts are not nodules within the structural networks of theories (an assumption widely generalized from inherited conception) but cognitive tools of scientific modeling belonging to scientific agents. Interestingly, their meaning does not depend on their deductive connection with observable statements or on providing different ways of using their content, but on their specific capacity to solve scientific problems by creating and manipulating models.

In this model-centric environment, concepts are characterized not as inert anachronistic entities, but as rich cognitive tools that originate through creative processes (in fact, the author emphasizes the creativity of these conceptual processes) and evolve historically. As Nersessian (2008) argues, this 'life' of concepts is not a mere diachronic transition of concepts in different theoretical contexts (regardless of whether their content depends on theories or not) but a complex socio-cognitive process in which multiple and diverse tools of scientific modelling and representation are involved. In our particular case, the meaning of the thermodynamic concept of 'entropy' would consist from a Nersessian-like perspective in its capacity for the expert agents who possess it to build effective thermodynamic models that quantitatively capture the degree of irreversibility during a certain interval in the evolution of a thermal system as a function of the amounts of heat emitted and the absolute temperature of the system. In fact, Clausius originally coined this concept (which emerges as a state function of an individual physical system) to reason analogously about the plausible mechanical significance of heat. ${ }^{7}$ One of the main advantages of this cognitive-historical perspective on scientific concepts is its powerful capacity to explain not so much what concepts are (i.e. scientific modeling tools), but above all what an agent's mental access to a given concept consists of and how it is made explicit in the modeling process.

\section{An Inferential View on the Meaning of Theoretical Concepts in Physics}

Having pointed out the most reliable current perspectives in philosophy of science about theoretical concepts, in this section we will

${ }^{7}$ See Sklar 1993. 
proceed to outline the basic part of our inferential theory that concerns largely the meaning of such concepts. First, we will start from Peacocke's thesis (1992) (later developed in proposals such as Wilson's 2006) by which the possession of a concept would be defined as the acceptance or cognitive assimilation of inferential rules by an expert epistemic agent within a practical context. However, the notion of 'rules' is highly problematic insofar as it places us directly in a 'playful' or 'performative' framework centered on the acts of agents; therefore, we would prefer to use the neutral term 'procedure' or even 'connection'. As Kindi and Arabatzis (2008) point out, the question of 'what are the concepts' (as well as their meaning and reference) and 'how do we access them' are two substantially different questions no matter how much we try to make one dependent on the other: we will face the first one directly. Assuming these methodological preliminaries, the theoretical concepts as such and without considering the particular mechanisms of access or possession of the agents, could satisfactorily be understood as a configuration of inferential procedures between different conceptual and formal resources in a concrete theoretical field.

This proposal of concepts as configuration of inferential procedures or connections is related to what Wilson (2006) calls 'patches' in his post-classical (or in other terms, 'semantic externalist') perspective of the theoretical concepts, which are nothing but convoluted inference-driven paths of significant information about a reality. Interestingly, the 'inferentialist' proposal that we will defend here regarding theoretical concepts in physics would be highly akin to Brandom's 2000 inferential proposal regarding concept semantics within the philosophy of language. Although we are remotely inspired by the above-mentioned drawing of the received view of a concept as a node within the theoretical semantic web (on which depends the notion of 'theoreticity' as a semantic dependence of the content of a concept in a theory, ${ }^{8}$ we will strongly reject, on the one hand, the (a) identification between theories as systems of structures (logical formulas, set-theories or model-theories), and on the other, (b) the fundamental relationship between concepts and theories. For the sake of the argument that we defend here, we assume with respect to (a) a 'semantic-representational' characterization of scientific theories as sets of scientific representations (Suárez and Pero 2019) in a broad sense, wherein theories are effectively constituted by formulas, diagrams, images, models, and so on. We understand scientific

\footnotetext{
${ }^{8}$ See Andreas 2017.
} 
representations in turn by their capacity to be inferentially exploited à la Suárez (2004); and at the same time — with respect to (b) — we assume that the fundamental relationship is not between concepts and theories, or concepts and models, but between concepts and scientific representations. However, we will leave the exhaustive defense of these assumptions for another future work.

It should also be remarked that we depart from a usual basic understanding of the distinction between 'theoretical concepts' and 'empirical' (or 'observational) concepts'. ${ }^{9}$ However, as we unfold our argument, the 'theoretical' characterization of a concept depends on what we can call 'meaningful content' within a representational context; just as the 'empirical' characterization (similarly to the operationalist proposal) depends on its 'meaningful content'. The theoretical-empirical dichotomy will have no explanatory relevance within our inferential proposal, since a concept may have both theoretical and empirical meaningfulness. For this reason, we will use the term 'theoretical concept' simply to point out that its semantic content is not directly inferentially connected with a set of relevant measurement operations, e.g. 'KELVIN TEMPERATURE'.

\subsection{Inferential Validity}

First, we must explain the idea of inferential validity, which will play a central role in our inferential proposal. To do so, we formulate the following criterion:

(IV) Criterion of Inferential Validity: An inferential extraction of information from a scientific representation ' $R$ ' constitutes an inferentially valid process if the content of the information obtained is derived in a syntactically (i.e. operationally) correct and semantically consistent manner from the content of the conceptual resources of $R$.

Preliminarily, it should be noted that this pragmatically-sensitive informal notion of 'inferential validity' generalizes the notion of 'logical validity' of deductive inferential systems (which is truth-preserving) to philosophically and scientifically interesting cases of inferences, whether inductive or hypothetical-abductive, based on real scientific practices. From this inferential framework, we understand the meaning of a concept in a representational context to be precisely the

${ }^{9}$ See Carnap 1966. 
particular contribution of the content of that concept to the validity of a plausible inference. In the same way, from this inferential account we could understand the reference of a concept in a representational context itself as the contribution to the fixation of the target phenomenon of the inferences that could be validly extracted from that representation.

To illustrate the validity of an inferential process, let us suppose that we are playing a game of clue, also known as 'cluedo'. ${ }^{10}$ In this context, let's imagine that player A concludes that the killer in this case is 'Colonel Mustard', since player B (who has the 'Miss Scarlett' card) has made a suspicious comment, and furthermore, this player B has a mustard stain from the hamburger that was eaten at dinner. Thus, the information $\beta$ (regardless of whether it is true or not) was obtained by player A through an invalid inferential process with respect to our (IV) in that $\beta$ is not actually derived (i) semantically from the content of B's card ('Miss Scarlett') nor (ii) syntactically from a correct operation (i.e. predicting the content of other players' cards) according to the game. On the other hand, $\beta$ is derived (iii) semantically inconsistently from the content (or lack thereof, rather) of 'a mustard stain', and (iv) an incorrect operation such as assuming that the player's elements have implications within the game. Explaining the analogy, the game clue would constitute the scientific representation $\mathrm{R}$ on which relevant information about the objective phenomenon (a murder) is obtained, players A and B would constitute the scientific agents, the cards ('Miss Scarlett' and 'Colonel Mustard') the conceptual resources ${ }^{11}$ of R.

The proper function of our (IV) is not only to offer an epistemic guarantee of the knowledge obtained in a context of scientific practice $^{12}$ but especially to establish a minimum framework on which to analyze how the conceptual components of a representation can contribute to obtaining epistemically robust knowledge. Let us suppose that $\mathrm{B}$ infers that the character of player A did not commit the crime from the fact that he himself has the 'MURDERER' card. In this scenario, the content of the concept-card 'MURDERER' would contribute to the validity of such inference, since what it denotes has

${ }^{10}$ For those who do not know, clue is a game for three to six players (each playing the role of a suspect in a crime) which consists of determining (i) who was the author of a murder, (ii) in which room of the mansion it occurred and (iii) with what murder weapon.

${ }^{11} \mathrm{By}$ 'conceptual resources' we mean the pieces of a representation whose content can be inferentially exploited to obtain relevant information about the phenomenon.

${ }^{12}$ This is a topic we will not go into for reasons of extension. 
been employed by means of a correct operation (i.e. conjecture based on the cards) by $\mathrm{B}$ to obtain relevant knowledge about the event. However, if we move to the case of scientific practices, we can see how the content of the theoretical concepts of the empirical natural sciences (in our case, thermophysics) contribute in different ways to draw valid inferences. Here I argue that these forms of contribution can be grouped into two substantially different modes of meaning according to their role within representational practices: empirical meaningfulness and theoretical significance. We will now go on to explain each of these modes in detail.

\subsection{Empirical Meaningfulness}

On the one hand, we find what we might call the "empirical meaningfulness ${ }^{\prime 13}$ of a theoretical concept. Before explaining in this sense of a theoretical physical concept, we will display in the following criterion (EM) the conditions under which the content of such concept contributes in an empirically meaningful way to the validity of an inference:

(EM) Criterion of Empirical Meaningfulness: A theoretical physics concept ' $\mathrm{C}$ ' will be empirically meaningful if its semantic capacity to consistently encode the data of experimentally measurable properties contributes to the fact that a plausible extraction of relevant information from a representation ' $R$ ' will be an inferentially valid process.

Firstly, the semantic capacity of a theoretical physical concept such as 'KELVIN TEMPERATURE' to encode the data derived from a set of measurement operations is determined, in this particular case, by how it orders that set of data (e.g. $155^{\circ}, 168^{\circ}$, and $175^{\circ}$ ) on a graduated scale that allows comparison. This data encoding derived from 'KELVIN TEMPERATURE' will be consistent if the encoding mechanisms do not vary depending on the objective phenomenon on which the measurement practices are performed. This is possible because this concept presupposes a fixed reference point on its scale, called 'absolute temperature' (Chang 2004), which allows unifying the thermal encoding mechanisms independently of the substance.

${ }^{13}$ Although we will use 'meaning' and 'significance' quite interchangeably in this paper, the word 'meaningfulness' will be taken to denote empirical content while 'significance' will denote conceptual content. 
So, how does this capability contribute to the validity of an inference? Suppose that an agent C infers from the result of a thermometric operation that a gaseous substance ' $\mathrm{g}$ ' has cooled down, going from $155^{\circ} \mathrm{K}$ at $\left(\mathrm{t}_{0}\right)$ to $148^{\circ} \mathrm{K}$ at $\left(\mathrm{t}_{1}\right)$. Thus, the ability to consistently encode thermometric values derived from the concept 'KELVIN TEMPERATURE' contributes in an empirically meaningful manner to the valid inference by $\mathrm{C}$ that the gaseous substance has cooled in the interval $t_{0}-t_{1}$. On the other hand, if this encoding capacity had not been consistent (suppose it varied according to the day of the week), then this information inferred by $\mathrm{C}$ would not have been valid, even if it were true. The reason is that, according to (IV), this information would not depend (semantically consistent and syntactically correct) on $155^{\circ}$ and $148^{\circ}$ having been obtained according to the empirical meaning of 'KELVIN TEMPERATURE', but on, for example, $155^{\circ}$ having been obtained on Sunday and $148^{\circ}$ on Tuesday.

Our criterion of empirical meaningfulness would also apply to concepts that are not directly connected to measurement processes, such as 'CLASIUS ENTROPY'. Unlike the previous case, the ability of this theoretical physical concept to encode data from thermometric measurements depends on its inferential connection with the concept 'KELVIN TEMPERATURE'. This inferential connection can be expressed as follows: the empirically meaningful way in which 'CLASIUS ENTROPY' contributes to the validity of a plausible inference (for example, that an agent $\mathrm{D}$ calculates that the change in entropy of $1 \mathrm{~kg}$ of water if it is heated from $303^{\circ} \mathrm{K}$ to $353^{\circ} \mathrm{K}$ is precisely $0.64 \mathrm{~kJ} / \mathrm{K}$ ) depends semantically and syntactically on the thermometric encoding capability of 'KELVIN TEMPERATURE'. Therefore, if this capacity were inconsistent, then the contribution of the content of 'CLASIUS ENTROPY' to the validity of the inference of agent D would no longer be empirically meaningful.

Finally, we argue that the essential role of this empirically meaningful contribution of a theoretical concept to the validity of agents' inferences in scientific practices is the causal connection between the content of such concept and the objective properties referred to by it in a representational context. In our case study it is the temperature of a gas itself that physically causes a thermometer (whose encoding capacity is derived from 'KELVIN TEMPERATURE') to indicate the value ' $148^{\circ}$ ', which in turn 'semantically causes' our agent C to validly infer that the temperature of the gas has fallen. In the following section we will go deeper into this issue. 


\subsection{Theoretical Significance}

On the other hand, the meaning of a theoretical concept can be not only empirical, but also 'theoretically significant' or just 'significant' respect to a given theoretical field, for example thermodynamics and statistical mechanics (or thermal physics in general), in which the scientific representation from which the employed concept is inserted. Again, we establish a criterion that sets out how the content of a concept can contribute in a theoretically significant way to the validity of actual scientific inferences:

(TS) Criterion of Theoretical Significance: A concept ' $C$ ' will be theoretically significant if the coherence of its semantic content (with respect to the content of other concepts within the same theory) contributes to the fact that the extraction of relevant information from a representation ' $R$ ' is an inferentially valid process.

First, the coherence of the content of a theoretical concept such as 'CLASIUS ENTROPY' with respect to other concepts included in the same representational context $\mathrm{R}$, is a semantic-relational property that would allow an agent to inferentially exploit the semantic content of such concepts in a unified manner and by virtue of their belonging to the same theoretical field, in this case that of thermodynamics. Thus, the semantic coherence between the concept 'CLASIUS ENTROPY' and the concept 'HEAT FLOW' contributes to an agent E inferring after observing a warm cup and a puddle of water, by virtue of the precepts of thermodynamics and even without quantitative procedures, that the heat has flowed from the hot cup to the block of ice with which it was in contact, and not just the other way around.

So, how precisely does the semantic coherence of the concepts of a representation contribute to the validity of this type of inference? Our answer to this question would be based on the fact that the inferred information (i.e. that heat has flowed from the cup to the ice block) depends on the syntactic procedures and the semantically coherent content of the concepts of 'CLASIUS ENTROPY' and 'HEAT FLOW', precisely because while the first one delimits (i) that heat can only flow spontaneously from a substance of higher to another of lower temperature, the second (ii) specifies that heat is a thermal form of energy of the objects (cups and ice blocks) definable from their temperature. It is precisely this close interrelationship between the 
content of both concepts that makes it possible for agent $\mathrm{E}$ to abductively infer in a procedurally correct and semantically consistent fashion within a thermodynamics representation, that the thermal energy that has caused the ice to melt came from the previously hot cup.

Note that no concept can simply be 'theoretically significant', since this would mean that its content is semantically coherent with the concepts of any theory, which is nonsense (or even worse: a triviality). On the contrary, each scientific concept may be significant with respect to a particular theory. In this sense, in what way does 'CLASIUS ENTROPY' constitute a thermodynamically significant concept? This concept is thermodynamically significant, first, because its content is semantically coherent with the main conceptual elements of that theoretical discipline, ${ }^{14}$ i.e. 'KELVIN TEMPERATURE', 'HEAT FLOW', 'HEAT ENGINE' and so on. Consequently, 'CLASIUS ENTROPY' is thermodynamically significant as it provides a high degree of semantic coherence with thermodynamic concepts incorporated into a representational context, which substantially contributes to an agent being able to validly infer relevant information about real thermal phenomena, such as in the case of the cup and the ice block.

In short, we defend that this theoretically significant contribution of a physical concept to the inferentially valid obtention of relevant information rely precisely on the fundamental epistemic role that scientific concepts have within representational practices that constitute a theoretical context. As we will see below, that a concept is theoretically (i.e. thermodynamically) significant also implies that the concept refers to its objective phenomenon through particular reference-fixation mechanisms.

We conclude this section by pointing out that empirical meaningfulness and theoretical significance are two different modes that integrate the same sense of theoretical concepts. This strictly philosophical distinction is particularly useful for the inferential analysis of the semantic behavior of theoretical concepts in the context of physics

\footnotetext{
${ }^{14}$ The issue of what are the main concepts of a theory will have to be addressed in a future work for reasons of extension. A short preliminary answer would be that every theory presupposes a certain spatial-hierarchical structure of concepts in which the main ones are those that contribute in a quantitatively and qualitatively greater way to the inferential obtention of information in the practical contexts delimited by that theory. For example, 'HEAT FLOW' will be a main concept (or more central, in gradual terms) of thermodynamics while 'METAL ENGINE' will not, precisely because the content of the former (but not the content of the latter) would be somehow presupposed in virtually all inferences made within this field.
} 
(particularly in our case of study, thermal physics) due precisely to the strong empirical-theoretical hybrid nature of these disciplines. As we shall now see, this distinction also plays a central role in the development of an inferential proposal about the referential mechanisms underlying these concepts.

\section{An Inferential View on the Reference of Theoretical Concepts in Physics}

In this second part of our inferential account we are going to analyze how theoretical concepts can fix their reference to real phenomena by virtue of their empirical meaningfulness and theoretical significance. Throughout the evolution of the philosophy of language in the twentieth century, various theories about the reference of proper names and other expressions have flourished, even finding application in the particular context of theoretical concepts (Bartels 2010; Psillos 2012), the two main ones being the descriptive and causal theories.

\subsection{Descriptivist and Causal Accounts on the Reference of Theoretical Concepts}

Firstly, the descriptive theory of reference (whose predominance was undefeated until the 1980s) applied to theoretical concepts in the physical sciences would roughly state that the reference of a particular concept is always fixed within a description or set of descriptions in which that term is inserted. Although from the classical descriptivism à la Frege-Russell or the improved one a satisfactory history can be given about how theoretical concepts are able to refer, it is not possible to explain how the same concept in two different theoretical contexts can come to refer to the same phenomenon, as long as (i) theories generate different descriptions, and (ii) the concept of a concept is description-dependent. In this sense, the concept 'BOLTZMANN ENTROPY' would refer to different properties if we place it within thermostatistical descriptions or within spin-statistical descriptions, where we also consider the spin of the molecules that constitute a system. Such a conclusion would not only be highly counterintuitive but also flagrantly false. However, the main problem of descriptivism comes from its combination with semantic holism (the meaning of a term is just a function within the semantic web of a theory) defended mainly by the historicist current of philosophy of science of Kuhn or Feyerabend. As pointed out by Psillos (2012), the combination of both theses implies that a minimum variation of the semantic web in which a concept is inserted would radically change 
its reference, even when the historical development of the discipline indicates that there is no such radicality in the reference of its terms.

Secondly, the causal theories of reference, by which the reference of a theoretical concept remains, on the one hand, fixed (in a causally oblique perceptive process) $)^{15}$ through the original baptism of the term; and on the other hand, causally transmitted from competent agent to competent agent during its historical evolution and that of the disciplines in which it is employed. For this reason, it would be possible in principle to give an explanatory account of what we can call 'inter-theoretical co-referentiality', that is, of why a concept can refer to the same phenomenon even when it is situated in two different theoretical contexts, either at the same time or during a period. On the other hand, the causal theory of reference would be highly deficient in explaining how concepts such as 'PHLOGISTON' or 'ETHER' (relevant in certain physical theories during a delimited historical period) fail to refer to their phenomena, almost trivializing the semantic conditions by which a concept refers.

\subsection{A Causal-Representational Inferential Account on the Reference of Theoretical Concept}

At this point in our analysis, we argue that causal and descriptive theories of reference based on the empirical meaningfulness and theoretical significance of a concept can satisfactorily converge into a unified causal-descriptive account to remedy the explanatory deficiencies of both approaches. This 'hybrid' account on conceptual reference was originally defended by Nola (1980) and later developed by Psillos (2012), where it is stated that its causal component guarantees the inter-theoretical referential stability of the concepts and its descriptive component allows the delimitation of the target phenomenon (later we will detail how this is possible). Recently, Bartels (2010) and Hoefer and Martí (2020) have also pointed out the explanatory effectiveness of descriptive causal theories in accounting for the reference fixing mechanism and the conceptual dynamics of scientific disciplines.

From our inferential framework, the reference of a concept within a scientific description or representation is the 'inferential target' or the target phenomenon on which information is inferentially obtained. Before continuing, we must clarify that according to our hybrid causal descriptivist theory, the reference of a concept is not

\footnotetext{
${ }^{15}$ Here a story is presupposed by which the community of experts perceives in person the baptismal event by which the reference of a concept is fixed.
} 
fixed only within a description or set of them, understanding 'description' as a logical or linguistic statement based on formulas, but within a vast plurality of well-defined semantic vehicles, i.e. linguistic descriptions, mathematical formulas, diagrams, conceptual schemes, detailed images, etc. Having clarified this point, we are now going to argue for the following core thesis: the reference of a theoretical concept within a representational context depends on both the empirical meaningfulness and the theoretical significance of its meaning (see section 3), these two understood as providing different referencefixing mechanisms.

\subsection{Causal Reference Fixation from Empirical Meaningfulness}

On the one hand, how the empirical meaningfulness of a theoretical concept contributes within a representational context (see section 3.2) to fix its reference or inferential target has to be partially understood from a causal perspective. However, against the main causal-centric theorists of reference, we defend that the key causal mechanism cannot be found in the socio-historical transmission of the conceptual reference but within the inferential-validity transmission chain. That chain is what we should call "empirical meaningfulness scaffolding' by which the target phenomenon referred to by the concept causally affects the semantic content of that concept, not in an experimentally direct fashion but in a way that is mediated by other empirically meaningful concepts such as 'KELVIN TEMPERATURE' or 'BOLTZMANN CONSTANT'. As can be seen in section 3.2. this 'causality' must be properly understood in two substantially different ways. On the one hand, it is the phenomenon that physically causes the experimental detector (i.e. a thermometer) to indicate a certain temperature value. On the other hand, it is this temperature value which would "semantically cause"16 (that is, it causes by virtue of its meaning) that after being encoded in a new theoretical field, this can be exploited in an inferentially valid way by an agent to obtain information on the temperature of the very same phenomenon that would physically cause a mark on the thermometric detector.

Illustratively, the empirical meaningfulness of "BOLTZMANN ENTROPY', a statistical mechanical reformulation (mathematically encoded in the famous $\mathrm{S}_{B}=\mathrm{k}_{B}$ In $\mathrm{W}$ ) of Clausius thermodynamic concept, depends directly on its semantic coherence with the statistical mechanical concept of 'BOLTZMANN CONSTANT', which inferentially

\footnotetext{
${ }^{16}$ This idea of 'semantic causation', wherein $\mathrm{X}$ causes $\mathrm{Y}$ in virtue of the meaning of X, was recently developed by Zhong (2015).
} 
relates the directly observational and consistently measurable content of 'KELVIN TEMPERATURE' (in whose thermometric operations the target phenomenon causally intervenes) with the statistical mechanically significant content of 'AVERAGE MOLECULAR KINETIC ENERGY'. Thus, the statistically significant mechanical content of 'BOLTZMANN CONSTANT' allows (identifying the temperature of a substance with the kinetic energy of its molecules) that the content of 'KELVIN TEMPERATURE' contributes in an empirically meaningful way to the validity of the inferences that an agent can draw within a statistical mechanical representational context. For example, the fact that an agent can infer that the entropy generated when manipulating the molecules of a gas is $0.69 \mathrm{~J} / \mathrm{K}$ in a valid fashion depends syntactically and semantically on (i) that $\mathrm{K}$ can be consistently re-encoded in molecular terms (statistically significant mechanics) thanks to 'BOLTZMANN CONSTANT', and (ii) that the experimentally measurable values of $\mathrm{K}$ are consistently encoded (section 3.2).

Once we have established these elements, we argue that the empirical meaningfulness of a concept provides a robust causal mechanism that would fix its reference, even under different representational-theoretical context. Following our example, this referencefixing mechanism would be grounded on (i) the fact that the actual temperature of the gas physically causes the thermometer to dial $1,380 \times 10^{-19} \mathrm{~K}^{-1}$, (ii) that $1.380 \times 10^{-19} \mathrm{~K}^{-1}$ semantically causes the value $0.69 \mathrm{~J} / \mathrm{K}$ of 'BOLTZMANN ENTROPY' to be empirically meaningful in a valid (statistical mechanical) inference, which allows (iii) the concept 'BOLTZMANN ENTROPY' encoded in the value $0.69 \mathrm{~J} / \mathrm{K}$ to refer to a real phenomenon linked to the temperature of the gas. The robustness of this causal mechanism of referencefixing is manifested in the fact that the reference of the concept 'BOLTZMANN ENTROPY' via $0.69 \mathrm{~J} / \mathrm{K}$ can be experimentally evaluated and tested, precisely because a property (temperature) of this referred phenomenon would physically cause the thermometer to mark $1.380 \times 10^{-19} \mathrm{~K}^{-1}$, which in turn causes semantically the value of $0.69 \mathrm{~J} / \mathrm{K}$.

Interestingly, this derived causal mechanism that we have just described and exemplified allows us to give a solution to the problem (typical of descriptivism plus semantic holism), ${ }^{17}$ of the variation of the reference of theoretical concepts in different theoretical contexts. The reason is that the reference of 'BOLTZMANN ENTROPY' will be the same from the field of thermophysics as from ferromagnetism,

${ }^{17}$ See Psillos 2012. 
precisely because its causal mechanism of reference-fixing will depend semantically-operatively on the same thermometric procedure of consistent encoding of experimental values. Our inferential explanation is that the empirically meaningful contribution of "BOLTZMANN ENTROPY' to the validity of the inferences does not vary according to the representational practice in which it is inserted, this concept refers to the same thermal phenomenon whether the agent $\mathrm{F}$ uses it to obtain information (i) about an adiabatic process in a liquid, or (ii) the disposition of the components of a magnetized iron bar.

\subsection{Representational Reference Fixation from Theoretical Significance}

On the other hand, we argue that from the theoretically significant way in which the content of a concept contributes to inferential validity, a descriptive (or properly 'representational') mechanism would be derived by which the reference of such concept would be precisely fixed. It is precisely the semantic coherence of the content of a concept with the content of other concepts in the same representation that makes it possible to delimit in a theoretically significant way the referential target on which relevant information is to be validly inferred.

For instance, the meaning of 'BOLTZMANN ENTROPY', ${ }^{18}$ which depends directly on the fact that it is semantically coherent with other statistical mechanical concepts, such as the 'MACROSTATE' (i.e. observable state of a molecular system) and the 'MACROSTATE' (i.e. microscopic deterministic configuration of a molecular system), will semantically/syntactically enable us to validly infer information about a statistical-mechanically referred phenomenon. Assuming that we depart from a statistical mechanical representation $\mathrm{R}$ of a gas, the semantic coherence of 'BOLTZMANN ENTROPY' and other statistical mechanical concepts in $\mathrm{R}$ provides a representationbased reference fixing mechanism, in the sense that this concept will precisely and accurately refer to a ratio of dissipated energy (measured in Joules) and absolute temperature (thermometrically measured in Kelvin degrees) which would be proportional to a set of observationally-indistinguishable microscopic configurations of the molecules of that gas.

Comparatively, while the content of 'CLAUSIUS ENTROPY' makes it possible semantically and syntactically for an agent $G$ to validly infer that the entropic change (understood as the phenomenon to

${ }^{18}$ See Callender 1999. 
which the concept refers $)^{19}$ that occurs when $1 \mathrm{~kg}$ of water is heated from $303^{\circ} \mathrm{K}$ to $353^{\circ} \mathrm{K}$ will be $0.64 \mathrm{~kJ} / \mathrm{K}$, the content of 'BOLTZMANN ENTROPY' allows the same agent $\mathrm{H}$ to validly infer with a much higher level of mechanical statistical precision that the value of entropy change will be $0.64582394 \mathrm{~kJ} / \mathrm{K}$. The precise reason is that, while the reference in the first case depends only on how agent $\mathrm{G}$ infers from a thermodynamic representation of the macroscopic values of the gas, the reference in the second case depends precisely on how agent $\mathrm{H}$ infers from a statistical mechanical representation of the observable values of the gas from the properties of its molecular components. Therefore, the representations in which the concepts are inserted provide them (based on the semantic connections with other concepts) with different mechanisms that set their reference with different degrees of precision depending on the theoretical context in which they are located.

Finally, let us point out that the main problem of causal theories (i.e., how a concept such as 'PHLOGISTON' ceases to have a reference in another theoretical context) would be solved in our inferential framework by means of the representational and causal mechanisms of reference fixation. In the paradigmatic case of 'PHLOGISTON', ${ }^{20}$ it would not simply cease to refer, but its referential target would be well-delimited or specified in a chemically significant fashion with the advent of modern chemistry in the nineteenth century. This is because its empirically meaningful contribution to the validity of inferences about what happens in combustion processes (i.e. the abduction that in combustion processes a substance is emitted) would take on a new chemical significance in the sense of the concept 'OXYGEN', which allows valid inferences to be made from the same experimental results that in combustion a substance would not be generated, but consumed, namely oxygen.

As we have tried to defend in this section, each one of the modes of meaning of a theoretical concept constitutes different inferential mechanisms of causal-descriptive or causal-representational fixation of the reference of that concept. On the one hand, the empirical meaningful contribution of a theoretical concept to the validity of an inferential concept constitutes in itself an inferential mechanism

\footnotetext{
${ }^{19}$ In a close way to Kroon's proposal (1987), the validity of the inferences involving the meaning of a concept constitutes a robust epistemic guarantee that the concept effectively refers within a representation to its intended target property.

${ }^{20}$ See Bartels 2010.
} 
that causally fixes the reference of this concept, granting it referential stability against its placement in different theoretical-representational contexts. In this way we would give a solution to the problem that we find in historicist proposals of science (i.e. Kuhn or Feyerabend) of the continuous referential variation of concepts, derived from subscribing to both descriptivism and semantic holism (Psillos 2012). On the other hand, the theoretically significant contribution of a theoretical concept to the validity of an inference supposes an inferential mechanism that fixes representationally the reference of such concept, providing referential precision to concepts according to their theoretical-representational context. This, unlike causal theories, would explain how the reference of concepts such as 'PHLOGISTON' did not disappear, but rather it was accurately refined (i.e. combustion processes) when placed within a new theoretical context such as modern chemistry. Therefore, our inferential proposal of the reference of theoretical concepts poses a solution to the main problems we find in descriptive and causal theories precisely through the integration of causal and representational mechanisms of the reference of counts, derived from our analysis of their empirical meaningfulness and theoretical significance, respectively (section 3). Next, let us see the clarifying potential of our inferential account in its application to the main concepts of entropy.

\section{Case Study: Assessing Entropy Concepts from an Inferential Perspective}

Once we have detailed our inferential framework on theoretical concepts, integrating both (i) a theory about the empirical meaningfulness and theoretically significant nature of their meaning and (ii) a descriptive causal theory about their reference, it could now be applied in the particular case of the different concepts of 'entropy' that we can find in different disciplinary fields to show their clarifying capacity and explanatory power. As Wicken pointed out:

The concept of entropy has had a long and interesting history, beginning with its implicit introduction by Carnot to its explicit formalization as a state function by Clausius to its statistical treatment by Boltzmann and Gibbs to its application to communication theory by Shannon. The latter achievement has seemed to several scientist a true generalization of the entropy conception, its freeing from the particular disciplinary framework of thermodynamics for application to probability distribution generally. This mistaken belief [...] (1987, p. 177) 
The rigorous and exhaustive evaluation of concepts of entropy (or the concept of entropy, as insisted by some authors such as Wicken above) has been the object of great controversy not only within the physical sciences, but also within the systematic philosophical reflection on thermal physics, consolidated from the foundational work of Lawrence Sklar (1993). Volumes have been written in the literature about this complex concept. However, no semantic analysis has been carried out so far about the conditions under which the different concepts of entropy can be really significant in real scientific practices: this is precisely our goal.

Up to this point in our analysis we have limited ourselves to using the theoretical concepts of thermodynamic entropy, originally developed by Clausius in 1865 to create a state function that served as a mechanical counterpart to heat, and Boltzmann's statistical mechanical entropy (whose standard formulation does not come from Boltzmann himself but from the Ehrenfests in 1911) as case studies (Sklar 1993). While our interest is focused on the domain of representational practices of the physical sciences ${ }^{21}$ we must also include the information theoretical entropy by the impact it has had in various fields of physics. That concept was developed by Shannon to quantify the uncertainty of the occurrence of a sequence of symbols in a communicative process. For reasons of extension, we will avoid the non-classical (e.g. von Neumann entropy) and eminently formal (e.g. KolmogorovSinai metric entropy) versions of entropy concepts. For a rigorous and exhaustive analysis of the enormous constellation of entropy concepts in various scientific fields and their diverse interrelations, see the 'conceptual map' outlined by Frigg and Werndl (2011).

\subsection{Clausius Entropy}

Preliminarily, it should be pointed out from our inferential framework that the content of 'KELVIN TEMPERATURE' ' $T$ ', on which the empirical meaning of all empirically meaningful entropic concepts rests, would be thermophysically significant (i.e. semantically consistent with the content of thermodynamics concepts) and has an observationally direct empirical meaningfulness when it comes to inferring thermal properties from an enormous variety of physical phenomena. On this notion of temperature, historically connected by Lord Kelvin with the so-called Third Law of Thermodynamics, the

\footnotetext{
${ }^{21}$ This rules out a plurality of concepts of entropy applied to the most varied fields, such as biology or even sociology.
} 
concept of 'CLAUSIUS ENTROPY' (which has played a central illustrative role in our argumentation) would be defined. The latter concept is expressed mathematically by means of ratios of infinitesimal heat flow and temperature values, where it only makes thermodynamic sense if expressed by ' $\mathrm{dS}$ ' intervals of entropy values.

During this paper we have defended from our inferential proposal that 'CLAUSIUS ENTROPY' possess an observationally indirect (since their content cannot be measured directly, there being no way to perform 'entropometric' operations) empirical meaningfulness, based on the consistent encoding of experimental values from thermometric operations. On the other hand, the content of this concept contributes in a thermodynamically significant way to the validity of the inferential obtaining of relevant information about thermophysical phenomena, due to the semantic coherence of its content with the main thermodynamic concepts: e.g. 'CLOSED SYSTEM', 'THERMAL EQUILIBRIUM' or 'STATE FUNCTION', among others. As noted in section 4, the fact that the content of 'CLAUSIUS ENTROPY' contributes in an empirically meaningfull and thermodynamically significant way to the validity of inferences in thermophysical practice contexts is derived, respectively, from (i) a causal mechanism that gives it referential stability in different theoretical-representational contexts, and (ii) a representational mechanism that establishes its reference from observable properties such as temperature or pressure.

Finally, from this inferential analysis of its modes of meaning and referential mechanisms we can understand the role of the concept 'CLAUSIUS ENTROPY' as an extremely useful representational tool for agents in thermodynamic practices when inferring from empirical data the degree of thermal irreversibility of certain processes.

\subsection{Boltzmann Entropy}

Barely a decade after Clausius coined his famous concept, Boltzmann sought to reformulate or even explain its content from the dynamic behavior (empirically inaccessible) of the molecules that made up the material substances through what we know today as Boltzmann entropy (Sklar 1993). We have argued previously that the theoretical concept 'BOLTZMANN ENTROPY' is empirically meaningful, since it is semantically connected (via an empirically meaningful scaffolding, see section 4.3) to the consistent encoding of thermometric values of 'KELVIN TEMPERATURE' and mediated by 'BOLTZMANN CONSTANT', thus giving a new statistical mechanical significance to the previous notion of 'KELVIN TEMPERATURE' in terms of the av- 
erage kinetic energy of molecular components of a system. Note that this empirical meaningfulness, unlike Clausius' notion, is mediated by the empirically meaningless content (due to the impossibility of obtaining experimental data about the speed and position of all molecules in a gas) of statistically significant concepts such as 'MICROSTATE'.

On the other hand, the mechanically significant statistical content of 'BOLTZMANN ENTROPY' contributes to agents being able to validly infer (calculate, abduct, predict and so on) in an extremely sophisticated way certain macroscopic properties of physical systems from the behavior of their microscopic properties, due to its complex representational or mechanical statistical mechanism of reference fixation. The fact that 'BOLTZMANN ENTROPY' has both empirical meaningfulness and statistical significance from a mechanical point of view suggests, from our inferential framework, that its content incorporates a causal mechanism that allows it to reference-fix (with great accuracy) in a stable way, regardless of whether an agent I uses this concept from the theoretical-representational context of gas kinetics or from fluid mechanics. In this sense, 'BOLTZMANN ENTROPY' refers in a stable and highly precise fashion to the values of an entropic property that belongs not to a physical system (as in the case of 'CLAUSIUS ENTROPY') but to a set of observationally inaccessible molecular configurations of that system.

The point is that, as Wallace (2012) states, this level of referential precision made possible by 'BOLTZMANN ENTROPY' implies an enormous difficulty not only mathematically (calculating an astronomical number of microscopic variables) but also technically (processing enormous amounts of molecular data) when using this concept in thermophysical practices related to realistic systems with a large number of components. Therefore, although the empirically significant and statistically mechanically significant content of "BOLTZMANN ENTROPY' allows agents to validly infer an enormous amount of relevant information about certain phenomena, the truth is that it also presupposes (precisely because of what Wallace points out above) an overwhelming amount of practical and computational resources to carry out such inferences.

\subsection{Shannon Entropy}

And last but not least, we find the notion of entropy famously developed by Shannon in the context of statistical analysis of message transmission processes, which was later to be called information the- 
ory. Although 'SHANNON ENTROPY' was developed within information theory, wherein it broadly measures the degree of 'uncertainty' caused by a received symbol, it has been constantly employed within the physics literature, mainly within the Jaynesian statistical mechanical formalism (Sklar 1993; Frigg and Werndl 2011). It should be noted that its mathematical expression (i.e. the logarithm of the probability of occurrence of a symbol) lacks any element that would allow formulating its content through explicitly physical quantities, such as temperature or pressure.

Initially, we can assess from our inferential framework that the content of 'SHANNON ENTROPY' cannot contribute in an empirically meaningful fashion to validly infer relevant information about any physically interesting phenomena precisely because its meaning cannot be semantically connected (even indirectly) to consistent thermometric operation, and therefore its content would not be causally connected with the phenomenon it is intended to refer to within a thermophysical representation. On the other hand, this concept would lack not only thermophysical significance but also physical significance in general, precisely because the concepts with which it is semantically coherent ('SENDER-RECEIVER', 'MESSAGE', 'TRANSMISSION CHANNEL' or 'NOISE') cannot be used for a competent agent to infer valid information about an actual physical system. Authors such as Wicken (1987) already warned of the profound semantic deficiency of Shannon entropy in contexts of representational practices in thermophysics.

Finally, that 'SHANNON ENTROPY' lacks both empirical meaningfulness and thermophysical significance would imply that it would not contribute semantically in any way to the validity of the inferences that can be drawn from thermophysical representations that incorporate it, which means that any epistemic success (correct predictions or satisfactory explanations) that such a representation may generate will be merely fortuitous. For example, Ben-Naim (2008) will use 'SHANNON ENTROPY' within a simple model of ideal gases (with a small number of molecules and no interatomic forces) to calculate or infer the value of entropy generated by the mixture of the components of two gases with different properties. Even if these numerical results coincide with those obtained from thermophysically significant concepts such as 'BOLTZMANN ENTROPY', their obtaining does not depend syntactically or semantically on the empirical character and thermophysical significance of such a concept, but on fortuitous factors linked to the processes of idealization and extreme 
abstraction in such a representational context. Therefore, in consequence of what is defended in our inferential framework, "SHANNON ENTROPY' would not have the semantic capacity to validly refer (without accuracy or precision) to physical phenomena.

\section{Conclusion: Toward an Integrated Inferential Account on Concepts and Representations}

In this paper we have defended an inferential proposal about the theoretical concepts in the field of physics, mainly based on the pragmatic notion of 'inferential validity', which refer to the fact that the piece of inferred information follows from another set of information in a semantically consistent and operationally correct fashion. Initially, we differentiate between (a) the empirical meaningfulness of a concept, which depends on a consistent encoding of experimental data; and (b) its theoretical significance, provided by its semantic coherence with theoretically-related concepts within the representational practice in which the concept is inserted. In this sense, we have also explained from our inferential frame how the different modes of meaning (or equivalently, the different conceptual contributions to the validity of inferences) of a concept, provide the semantic mechanisms by which a theoretical concept refers.

Additionally, from our inferential proposal we have developed a causal-descriptive (or properly causal-representation) account of the reference of theoretical concepts, similar to the one defended by Psillos (2012) or Hoefer and Martí (2020). On the one hand, the empirical meaningfulness of the content of a theoretical concept provides a robust causal reference-fixing mechanism, which helps to explain the problem of variation of the conceptual reference by anchoring it to the same consistent encoding of experimental values. On the other hand, the theoretical significance of a theoretical concept provides the descriptive (or more generally 'representational') component of the reference-fixing mechanism around such concept. From here we could explain how certain concepts (i.e. 'PHLOGISTON') do not lose their reference when a new theoretical context emerges, but rather their reference would be theoretically refined and fine-grained. Finally, we have used all the elements of our inferential proposal to carefully analyze the meaning and the reference mechanisms of the main concepts of entropy, undoubtedly one of the most problematic family of concepts in all the physical sciences.

Finally, we conclude our argument by emphasizing how one of the main benefits of our inferential framework is precisely its capacity to 
analytically clarify the semantic labyrinths that underlie not idealized scientific scenarios but actual scientific practices. After defending the explanatory virtues of our perspective regarding the meaning and reference of theoretical concepts, we will leave for future works the development of a proposal also inferential (similarly to Suárez 2004) about the semantic behavior of scientific representations based on their conceptual content and their formal resources. The key will then be to explore in greater detail the integral mechanisms by which the significant content of concepts contributes to the valid inferential exploitation of a plurality of representations: this will allow us to understand the subtle processes by which the conceptual richness of the scientific disciplines permeates from the diagram in an introductory textbook to the system of formulas on the blackboard of a Nobel Prize winner, contributing semantically and epistemically to their development. This is an onerous philosophically task, but one that will undoubtedly be worth pursuing. ${ }^{22}$

\section{REFERENCES}

Andreas, H., 2017, "Theoretical Terms in Science", en Edward N. Zalta (ed.), The Stanford Encyclopedia of Philosophy (Fall 2017 edition) $<$ https://plato.stanford.edu/archives/fall2017/entries/theoreticalterms-science/ $>$ [date of last consultation: 15/09/2020].

Arabatzis, T., 1995, The Electron: A Biographical Sketch of a Theoretical Entity, Dissertation, Princeton University.

Bartels, A., 2010, "Explaining Referential Stability of Physics Concepts: The Semantic Embedding Approach", Journal for General Philosophy of Science/Zeitschrift für Allgemeine Wissenschaftstheorie, vol. 41, no. 2, pp. 267-281.

Ben-Naim, A., 2008, A Farewell to Entropy. Statistical Thermodynamics Based on Information, World Scientific, Singapore.

Brandom, R., 2000, Articulating Reasons: An Introduction to Inferentialism, Harvard University Press, Harvard.

Bridgman, P.W., 1927, The Logic of Modern Physics, Macmillan, New York.

Callender, C., 1999, "Reducing Thermodynamics to Statistical Mechanics: The Case of Entropy", Journal of Philosophy, vol. 96, no. 7, pp. 348373.

${ }^{22}$ I particularly wish to thank the anonymous reviewers for their really useful comments and suggestions. This work has been funded by an FPU Grant (FPU16/0774) of the Spanish Ministry of Education. It has been developed under the research framework of LOGOS and the research group "Laws, explanation and realism in physical and biomedical sciences" (FFI2016-76799-P). 
Carnap, R., 1966, Philosophical Foundations of Physics: An Introduction to the Philosophy of Science, Basic Books, New York.

Chang, H., 2019, "Operationalism", in Edward N. Zalta (ed.), The Stanford Encyclopedia of Philosophy (2019 Edition), <https://plato.stanford.edu/ archives/win2019/entries/operationa $\operatorname{lism} />$ [date of last consultation: $15 / 09 / 2020]$.

- - 2004, Inventing Temperature: Measurement and Scientific Progress, Oxford University Press, USA.

Frigg, R. and C. Werndl, 2011, "Entropy - A Guide for the Perplexed", in Claus Beisbart and Stephan Hartmann (eds.), Probabilities in Physics, Oxford University Press, Oxford, pp. 115-142.

Hoefer, C., and G. Martí, 2020, "Realism, Reference and Perspective", European Journal for Philosophy of Science, vol. 10, no. 38.

Kindi, V. and T. Arabatzis, 2008, "The Problem of Conceptual Change in the Philosophy and History of Science", in Stella Vosniadou (ed.), Handbook of Research on Conceptual Change, Routledge, London.

Kroon, F.W., 1987, "Causal Descriptivism", Australasian Journal of Philosophy, vol. 65, no. 1, pp. 1-17. (doi:10.1080/00048408712342731)

Nersessian, N., 2008, Creating Scientific Concepts, MIT Press, Mass.

Nola, R., 1980, "Fixing the Reference of Theoretical Terms", Philosophy of Science, vol. 47, no. 4, pp. 505-531. (doi: 10.1086/288954)

Peacocke, C., 1992, A Study of Concepts, MIT Press, Cambridge, Mass.

Psillos, S., 2012, "Causal Descriptivism and the Reference of Theoretical Terms", in Athanassios Raftopoulos and Peter Machamer (eds.), Perception, Realism, and the Problem of Reference, Cambridge University Press, New York.

Shapere, D., 1985, "Reason and the Search for Knowledge", Philosophy of Science, vol. 52, no. 2, pp. 310-312.

Sklar, L., 1993, Physics and Chance: Philosophical Issues in the Foundations of Statistical Mechanics, Cambridge University Press, Cambridge.

Suárez, M., 2004, "An Inferential Conception of Scientific Representation", Philosophy of Science, vol. 71, no. 5, pp. 767-779.

Suárez, M. and F. Pero, 2019, "The Representational Semantic Conception”, Philosophy of Science, vol. 86, no. 2, pp. 344-365.

Wallace, D., 2012, "The Necessity of Gibbsian Statistical Mechanics", PhilSci archive. <http://philsci-archive.pitt.edu/15290/> [date of last consultation: 15/09/2020]

Wicken, J.S., 1987, "Entropy and Information: Suggestions for Common Language", Philosophy of Science, vol. 54, no. 2, pp. 176-193.

Wilson, M., 2006, Wandering Significance: An Essay on Conceptual Behavior, Clarendon Press, Oxford.

Zhong, L., 2015, "Semantic Normativity and Semantic Causality", Philosophy and Phenomenological Research, vol. 94, no. 3.

Recibido el 4 de agosto de 2020; aceptado el 31 de octubre de 2020. 